\title{
PGD management scheme for older females with balanced translocations: Do older females have less chance of balanced embryo transfer?
}

\author{
Pinar Tulay', Meral Gültomruk ${ }^{2}$, Necati Fındıklı ${ }^{2}$ Mustafa Bahçeci ${ }^{2}$ \\ ${ }^{1}$ Department of Medical Genetics, Near East University School of Medicine, Near East Avenue, Nicosia, Cyprus \\ ${ }^{2}$ Bahçeci Assisted Reproductive Technology Centre, Istanbul, Turkey
}

\section{Abstract}

Objective: Carriers of reciprocal and Robertsonian translocations have a higher risk of experiencing infertility and repeated miscarriages. It is well established that with advancing maternal age, the risk of aneuploidies in embryos increases. In this study, the chance of developing balanced embryos in translocation carriers with advanced maternal age was analyzed to establish a management scheme for couples seeking fertility treatment and preimplantation genetic diagnosis (PGD).

Material and Methods: Biopsy was performed on cleavage-stage embryos. Multicolor fluorescence in situ hybridization was used for PGD. The translocation carriers underwent a total of 55 cycles of PGD. Genetics diagnosis and cycle outcomes of PGD cases were examined.

Results: This study showed that the chance of obtaining a balanced embryo from the Robertsonian translocation carriers was significantly less when the maternal age is advanced. Similar rates for balanced embryos were obtained from the reciprocal translocation carriers.

Conclusion: The results of this study show that maternal age plays an important role and that genetic counselling and planning for a PGD cycle in translocation carriers, particularly for Robertsonian carriers, must be accordingly adapted. (J Turk Ger Gynecol Assoc 2016; 17: 91-5)

Keywords: PGD, maternal age, human embryo, translocation, PGD counselling

Received: 8 March, 2016 Accepted: 6 April, 2016

\section{Introduction}

Couples with known chromosomal abnormalities and structural changes opt for preimplantation genetic diagnosis (PGD) to avoid prenatal diagnosis or miscarriages. PGD is a method of selecting unaffected embryos from patients with translocations or single gene disorders. In humans, the incidence of structural changes, including translocations, is frequent $(1,2)$. Translocations are grouped in two categories: reciprocal, the most common form, and Robertsonian. Reciprocal translocations occur due to an exchange of two ends of non-homologous chromosomes, whereas the rearrangement of two acrocentric chromosomes arises in Robertsonian translocation carriers occurring in 1 in 900 live births (3). The phenotype of balanced reciprocal and Robertsonian translocation carriers is normal; however, they usually have a lower chance of successful natural reproduction $(1,4)$. Therefore, such couples opt for PGD to select a balanced embryo and increase their implantation and pregnancy rates. PGD is a highly invasive technique that necessitates in vitro fertilization followed by polar body or embryo (cleavage or blastocyst stage) biopsy. It is well established that there is a higher risk of aneuploidy in embryos with advancing maternal age (5). Additionally, advanced maternal age has been associated with higher levels of meiotic errors (6). Therefore, for these patients, genetic counselling holds great importance. These patients must be thoroughly informed about the risks of reproductive outcomes, and treatment strategies should be explained to these couples. In this study, we aimed to investigate if there was any effect of maternal age on the chromosomal status of embryos as well as preimplantation embryo development and to develop a management scheme for these patients.

\section{Material and Methods}

This retrospective study analyzed PGD results, the number of balanced embryos, and embryo transfer, followed by the analysis of pregnancy rates in carriers undergoing assisted reproductive technology treatments in Bahçeci ART Centre.

\section{Patient information}

Translocation carriers were subdivided according to maternal age ( $<35$ years and advanced maternal age of $\geq 35$ years) and translocation type, i.e., reciprocal and Robertsonian. A total of 20 couples with maternal ages of $<35$ years with 37 PGD cycles were analyzed within the reciprocal translocation carrier group. The advanced maternal age group of recipro- 
cal translocation carriers included 15 couples. Twelve couples with 17 PGD cycles were involved within the Robertsonian carrier group of maternal age of $<35$ years and three couples with eight cycles of PGD were in the advanced maternal age group. The couples undergoing PGD were counselled by an infertility specialist and medical doctors. PGD and limitations, including the risk of misdiagnosis due to mosaicism and technical errors, were explained to the couples. Prior to PGD cycles, the patients were informed about the procedure, and the potential risks were explained to and informed consent was obtained from each patient; ethical approval was granted. Chromosome banding techniques were used to obtain the karyotypes of all patients. The translocations of carriers were confirmed using fluorescence in situ hybridization (FISH). For FISH analysis, a total of two sub-telomeric probes and one centromeric probe were used.

\section{Controlled ovarian stimulation and embryology}

Stimulation treatment was applied for each patient as previously described (7). Briefly, to induce ovulation, human chorionic gonadotrophin (hCG) (5,000 IU; Ovidrelle; Merck Serono, UK) was injected, followed by the aspiration of follicles transvaginally after 35-36 h of the hCG injection. Hyaluronidase treatment was applied to oocytes following $2 \mathrm{~h}$ of culturing. Sperms were washed and prepared for the Intra-cytoplasmic sperm injection (ICSI) procedure by discontinuous colloidal silica gel gradient (PureSperm; Nidacon, Sweden). Only meiosis II (MII) stage oocytes were microinjected, and the oocytes were cultured for 16-18 $\mathrm{h}$ following ICSI in a 5\% $\mathrm{CO}_{2}$ and 5\% $\mathrm{O}_{2}$ incubator (INB203C; IKS International, Netherlands). Embryo scoring was assessed in terms of the number of cells and presence of even and uneven cells.

\section{Embryo biopsy and PGD}

Embryos were biopsied on day 3 of embryonic development $\left(\operatorname{Octax}^{\mathrm{TM}}\right.$, MTG; Munster, Germany). Embryos with less than six cells and more than $20 \%$ fragmentation were not biopsied. Three hundred eighty-nine embryos, of which 227 were from the reciprocal translocation carriers with maternal age of $<35$ years and 162 were from those with advanced maternal age, were biopsied. One hundred eighteen embryos, of which 131 obtained from the Robertsonian translocation carriers with maternal age of $<35$ years and 49 from those with advanced maternal age, were biopsied. Poly-L-lysine coated slides were used for FISH analysis, and one cell was analyzed for each embryo (Thermo Scientific; Darmstadt, Germany). Details of the translocations for each couple and the list of probes used are listed in Table 1. The poly-L-lysine coated slides were washed to remove excess probe hybridization as described by the manufacturer, and each nucleus was analyzed by counterstaining with $0.15 \mathrm{ng} / \mathrm{mL}$ of 4, 6-diamidino-2-phenylindole (DAPI, Medimiks; İstanbul, Turkey). Two expert scientists analysed the outcome of the PGD for translocations.

\section{Statistical analysis}

GraphPad prism v6 (GraphPad Software, Inc.; La Jolla, California, USA) was used for statistical analysis. The significance of obtaining balanced embryos in the translocation carriers with respect to maternal age was evaluated by the Chi-square test.

\section{Results}

Overall, $79 \%$ (1154/1457) of the oocytes collected matured to the MII stage, and 72\% (832) of these were normally fertilized with two pronuclei. Seventy four percent (619) of the normally developing embryos were biopsied for PGD (Table 2). Sixty agematched patients with no chromosomal rearrangements were analyzed for the developmental rate as a control group within the same time period. Ninety three percent (551/594) of the oocytes obtained from the control group developed to the MII stage, and 79\% (438) of these fertilized. The maturation and fertilization rates were similar between the two groups (Table 2). Overall, 63 of 389 (16\%) embryos from the reciprocal translocation carriers were chromosomally balanced, and eight of these were transferred, resulting in two pregnancies. The chances of obtaining balanced embryos from the reciprocal translocation carriers grouped in the maternal age $<35$ years $(16 \%, 38 / 227)$ and advanced maternal age $(15 \%, 25 / 162)$ groups were similar (Table 3 ). The number of balanced embryos was further analyzed according to the sex of the carrier. From 19 female carriers, 175 embryos were obtained. Of these, 14\% (24) were balanced. The chances of obtaining balanced embryos from male carriers were also at similar levels (18\%, 39/214).

In the Robertsonian translocations, a total of 187 embryos were biopsied, and $42 \%$ (78) of these embryos were shown to be balanced (Table 3). When the number of balanced embryos was further investigated according to the maternal age groups, considerably fewer balanced embryos $(30 \%, 17 / 56, \mathrm{p}<0.05)$ were obtained from the advanced maternal age group than from the younger patients who were $<35$ years $(45 \%, 59 / 131)$. Similar to the reciprocal translocation carriers, the number of balanced embryos obtained from the female $(38 \%, 35 / 92)$ and male $(45 \%$, 43/95) carriers was similar (Table 3 ).

\section{Discussion}

In this study, the role of maternal age in obtaining balanced embryos from translocation carriers was investigated. Overall, among the reciprocal translocation carriers, there was no difference in the number of balanced embryos between the advanced maternal age group and younger females. However, in the Robertsonian translocation carriers, it was observed that the number of balanced embryos was significantly decreased in the advanced maternal age group. Therefore, for the Robertsonian carriers, there may be an adverse effect of maternal age on PGD outcome. Advanced maternal age is well known to increase the risk of aneuploid pregnancy (8). This may be more noticeable in Robertsonian translocation carriers because the most commonly found aneuploidies in embryos include the same chromosomes that are also involved in Robertsonian translocations, such as chromosomes 13, 14, 15, 21 , and 22 (9-12). In the present study, the majority of embryos in Robertsonian translocation carriers involved chromosomes 13,14 , and 21 . Therefore, it is possible that the effect of maternal age on PGD outcome is due to the fact that these chromo- 
Table 1. Patient information list. Patient ID with maternal age at the time of oocyte retrieval, karyotype, and list of probes used in PGD analyses are listed. All probes are from Cytocell (UK) and Abbott Molecular Inc (USA)

\begin{tabular}{|c|c|c|c|}
\hline $\begin{array}{l}\text { Patient } \\
\text { ID }\end{array}$ & $\begin{array}{c}\text { Maternal } \\
\text { age }\end{array}$ & Karyotype & FISH probes used \\
\hline 1 & 39 & 46,XY, t(1;2)(p?36;p?14-16) & LPT 1p (Green), CEP 1 (Spectrum Orange), LPT 2p (Red) \\
\hline 2 & 41 & $46, \mathrm{XY}, \mathrm{t}(1 ; 2)(\mathrm{q} 42 ; \mathrm{q} 14.2)$ & LPT 1p (Green), LPT 1q (Red), TelVysion 2q (Spectrum Orange) \\
\hline 3 & 30 & $46, \mathrm{XX}, \mathrm{t}(1 ; 5)(\mathrm{q} 22 ; \mathrm{q} 23)$ & LPT 1q (Green), CEP 1 (Spectrum Orange), LPT 5q (Red) \\
\hline 4 & 30 & 46,XY, t(1;6)(q23.1;q21) & LPT 1q (Green), CEP 1 (Spectrum Orange), LPT 6q (Red) \\
\hline 5 & 40 & $46, \mathrm{XY}, \mathrm{t}(1 ; 9)(\mathrm{p} 32 ; \mathrm{q} 22)$ & LPT 1p (Green), LPT 1q (Red), TelVysion 9q (Spectrum Orange) \\
\hline 6 & 34 & 46,XX, t(1;10)(p?34;p11.2) & LPT 1p (Green), CEP 10 (Spectrum Aqua), LPT 10p (Red) \\
\hline 7 & 40 & $46, \mathrm{XX}, \mathrm{t}(1 ; 10)(\mathrm{p} 32 ; \mathrm{q} 21.3)$ & LPT 1p (Red), CEP 10 (Spectrum Aqua), LPT 10q (Green) \\
\hline 8 & 38 & $46, \mathrm{XY}, \mathrm{t}(1 ; 10)(\mathrm{p} 22 ; \mathrm{q} 22)$ & LPT 1p (Red), CEP 10 (Spectrum Aqua), LPT 10q (Green) \\
\hline 9 & 41 & $46, \mathrm{XY}, \mathrm{t}(1 ; 16)(\mathrm{q} 21 ; \mathrm{q} 12)$ & LPT 1q (Red), CEP 1 (Spectrum Orange), LPT 16q (Green) \\
\hline 10 & 39 & $46, \mathrm{XY}, \mathrm{t}(1 ; 22)(\mathrm{q} 12 ; \mathrm{q} 11.2)$ & LPT 1q (Red), CEP 1 (Spectrum Orange), LPT 22q (Green) \\
\hline $11^{*}$ & 36 & $46, \mathrm{XY}, \mathrm{t}(2 ; 5)(\mathrm{p} 11.2 ; \mathrm{q} 33)$ & LPT 2p (Red), LPT 2q (Green), LPT 5q (Red)** \\
\hline 12 & 31 & $46, X Y, t(2 ; 10)(q ? 23 ; p ? 13)$ & LPT 2q (Green), CEP 10 (Spectrum Aqua), LPT 10p (Red) \\
\hline 13 & 29 & $46, X X, t(2 ; 10)(q 36 ; q 22)$ & TelVysion 2q (Spectrum Orange), CEP 10 (Spectrum Aqua), LPT 10q (Green) \\
\hline 14 & 38 & $46, \mathrm{XX}, \mathrm{t}(2 ; 18)(\mathrm{p} 15 ; \mathrm{p} 11.2)$ & LPT 2p (Red), TelVysion 2q (Spectrum Orange), LPT 18p (Green) \\
\hline $15^{*}$ & 30 & $46, \mathrm{XY}, \mathrm{t}(3 ; 5)(\mathrm{q} 12 ; \mathrm{p} 12)$ & LPT 3p (Green), LPT 3q (Red), LPT 5p (Red)*** \\
\hline 16 & 33 & 46,XY, t(3;10)(p21.3;p15) & TelVysion 3p (Spectrum Green), CEP 10 (Spectrum Aqua), LPT 10p (Red) \\
\hline 17 & 32 & $46, \mathrm{XX}, \mathrm{t}(4 ; 10)(\mathrm{q} 31.2 ; \mathrm{q} 21.2)$ & TelVysion 4q (Spectrum Orange), CEP 10 (Spectrum Aqua), LPT 10q (Green) \\
\hline 18 & 33 & $46, X Y, t(5 ; 10)(q 13 ; q 24)$ & LPT 5q (Red), CEP 10 (Spectrum Aqua), LPT 10q (Green) \\
\hline 19 & 24 & $46, X Y, t(5 ; 11)(q 33 ; p 15)$ & LPT 5q (Red), CEP 11 (Spectrum Aqua), TelVysion 11p (Spectrum Green) \\
\hline 20 & 37 & $46, \mathrm{XX}, \mathrm{t}(5 ; 20)(\mathrm{q} 31 ; \mathrm{q} 13.3)$ & LPT 5q (Red), TelVysion 20p (Spectrum Green), TelVysion 20q (Spectrum Orange) \\
\hline $21^{*}$ & 33 & 46,XY, t(5;22)(q22;qter) & LPT 5p (Red), LPT 5q (Red), LPT 22q (Green), LSI 22 (Spectrum Green)** \\
\hline 22 & 25 & $46, \mathrm{XX}, \mathrm{t}(7 ; 10)(\mathrm{p} 13 ; \mathrm{p} 11.2)$ & LPT 7p (Green), CEP 10 (Spectrum Aqua), LPT 10p (Red) \\
\hline 23 & 28 & $46, \mathrm{XY}, \mathrm{t}(8 ; 22)(\mathrm{q} 23.2 ; \mathrm{qter})$ & LPT 8q (Red), CEP 8 (Spectrum Aqua), LPT 22q (Green), LSI 22 (Spectrum Green)** \\
\hline 24 & 34 & $46, X Y, t(9 ; 10)(p 13 ; q 11.2)$ & LPT 9p (Red), CEP 9 (Spectrum Aqua), LPT 10q (Green) \\
\hline 25 & 36 & $46, X Y, t(9 ; 12)(q 22 ; \mathrm{q} 24.3)$ & TelVysion 9q (Spectrum Orange), CEP 9 (Spectrum Aqua), LPT 12q (Green) \\
\hline 26 & 29 & 46,XX, t(9;12)(q22.3;q13.3) & TelVysion 9q (Spectrum Orange), CEP 9 (Spectrum Aqua), LPT 12q (Green) \\
\hline $27^{*}$ & 30 & $46, X X, t(9 ; 20)(q 34 ; q 13)$ & TelVysion 9q (Spectrum Orange), CEP 9 (Spectrum Aqua), TelVysion 20q (Spectrum Orange) \\
\hline 28 & 28 & $46, \mathrm{XX}, \mathrm{t}(10 ; 12)(\mathrm{q} 26 ; \mathrm{q} 24)$ & LPT 10q (Green), CEP 10 (Spectrum Aqua), TelVysion 12q (Spectrum Orange) \\
\hline 29 & 27 & $46, \mathrm{XX}, \mathrm{t}(10 ; 14)(\mathrm{q} 22.3 ; \mathrm{q} 13)$ & LPT 10q (Green), CEP 10 (Spectrum Aqua), LPT 14q (Red) \\
\hline 30 & 32 & $46, \mathrm{XX}, \mathrm{t}(11 ; 22)(\mathrm{q} 11.2 ; \mathrm{q} 13.3)$ & $\begin{array}{l}\text { TelVysion 11q (Spectrum Orange), CEP } 11 \text { (Spectrum Aqua), LPT 22q (Green), } \\
\text { LSI } 22 \text { (Spectrum Green)** }\end{array}$ \\
\hline 31 & 35 & $46, X X, t(13 ; 17)(q ? 14 ; q 21)$ & LPT 13q (Green), CEP 17 (Spectrum Aqua), TelVysion 17q (Spectrum Orange) \\
\hline 32 & 26 & $46, \mathrm{XY}, \mathrm{t}(13 ; 22)(\mathrm{p} 11.2 ; \mathrm{q} 13.1)$ & LPT 13q (Red), LPT 22q (Green), LSI 13 (Spectrum Orange), LSI 22 (Spectrum Green)** \\
\hline $33^{*}$ & 36 & $46, \mathrm{XY}, \mathrm{t}(16 ; 22)(\mathrm{q} 23.1 ; \mathrm{q} 13)$ & LPT 16q (Green), CEP 16 (Spectrum Aqua), LPT 22q (Green)** \\
\hline 34 & 31 & 46,XX, t(17;19)(q11.2;p13.3) & TelVysion 17q (Spectrum Orange), CEP 17 (Spectrum Aqua), LPT 19p (Green) \\
\hline 35 & 38 & $46, \mathrm{XY}, \mathrm{t}(19 ; 22)(\mathrm{p} 13.3 ; \mathrm{q} 11.2)$ & LPT 19p (Red), TelVysion 19q (Spectrum Orange), LPT 22q (Green) \\
\hline 36 & 35 & $45, X Y, t(13 ; 14)(q 10 ; q 10)$ & LPT 13q (Green), LPT 14q (Red), LSI 13 (Spectrum Orange) \\
\hline 37 & 39 & $45, \mathrm{XX}, \mathrm{t}(13 ; 14)(\mathrm{q} 10 ; \mathrm{q} 10)$ & LPT 13q (Green), LPT 14q (Red), LSI 13 (Spectrum Orange) \\
\hline
\end{tabular}




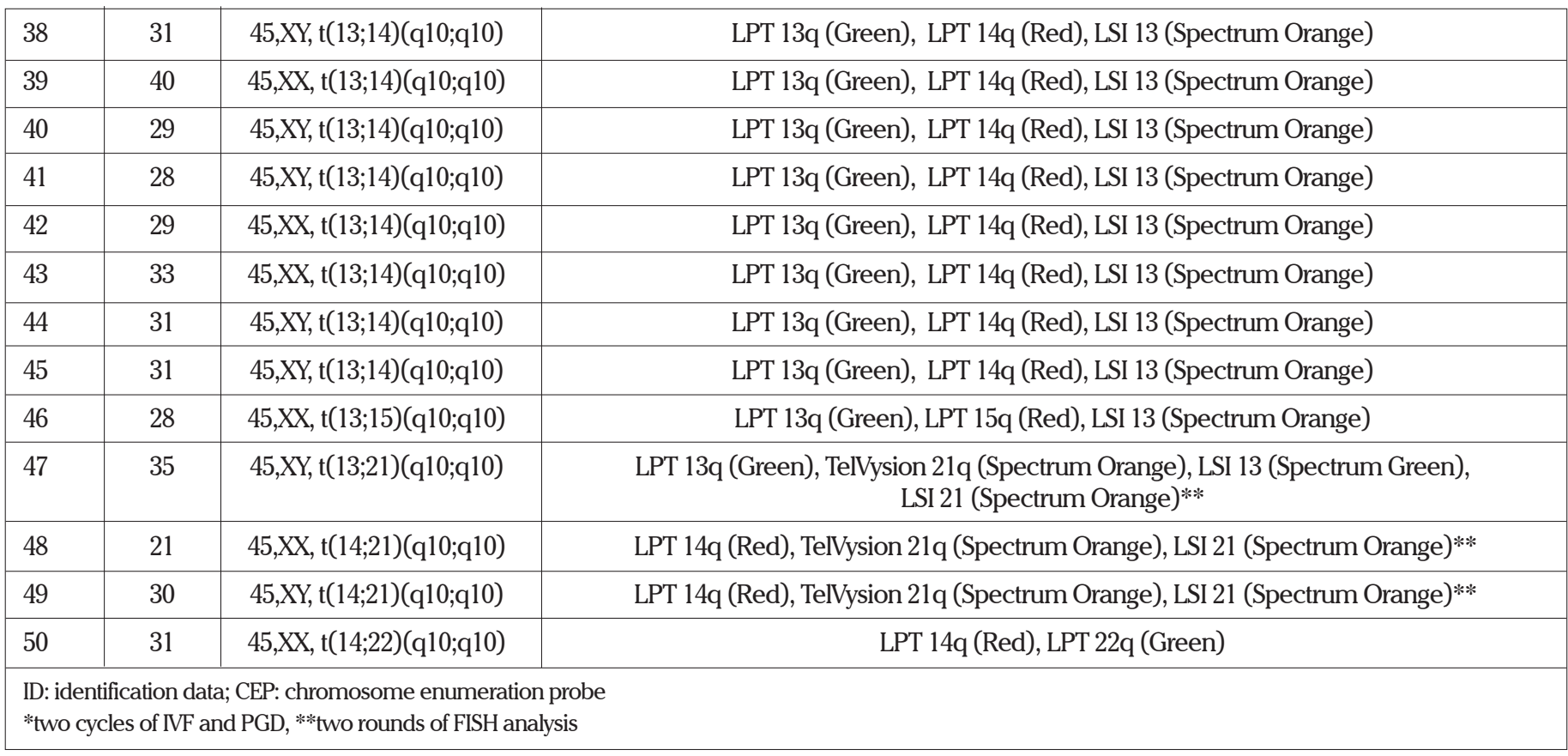

somes are the most commonly observed chromosomal abnormalities in embryos. Therefore, for the Robertsonian carriers, we cannot distinguish the segregation pattern of these chromosomes from aneuploidy that may be observed in non-carriers as well. The likelihood of obtaining a balanced embryo did not seem to be affected by the sex of the carrier. Similar findings have also been previously reported for reciprocal translocation carriers (8). These findings are particularly important to counsel patients prior to and during a PGD cycle. Understanding all these factors and the possible risks and outcomes of PGD are essential for effective counselling. Furthermore, evaluating couples' motives and considerations while opting for PGD would

Table 2. Table showing the number of patients, oocytes retrieved with the number of meiosis II (MII) stage oocytes, and biopsied embryos

\begin{tabular}{|l|c|c|}
\hline Number of patients & $\begin{array}{c}\text { Translocation } \\
\text { Carriers }\end{array}$ & $\begin{array}{c}\text { Control } \\
\text { Group }\end{array}$ \\
\hline Number of retrieved oocytes & 50 & 60 \\
\hline Number of injected oocytes (MII) & 1457 & 594 \\
\hline Number of biopsied embryos & 6154 & 551 \\
\hline
\end{tabular}

provide valuable information for improving genetic counselling and clinical care for these couples. Therefore, the results of this study provide significant information to establish a management scheme for translocation carriers undergoing PGD.

One of the drawbacks of this study was that we only analyzed the chromosomes involved in translocation. However, mitotic recombination of these chromosomes has been suggested to interrupt segregation of the normal chromosomes, leading to aneuploidies (13-18). Therefore, some aneuploid chromosomes that were not tested by FISH may have been missed. With the use of array-based comparative genomic hybridization (aCGH), translocation as well as aneuploid embryos would have been detected. However, FISH is still widely used to detect translocations $(19,20)$. This is mostly due to the size of breakpoints involved in translocation because in some cases, even the high-resolution aCGH cannot detect the translocated segment (21). Additionally, neither FISH nor aCGH could distinguish a balanced embryo from a normal one.

To conclude, advancing maternal age may adversely affect the chromosome complement in embryos. The chances of attaining a balanced embryo were significantly reduced in Robertsonian translocation carriers compared to those in younger females. Therefore, it is important to counsel carriers, particularly those with advanced maternal age, undergoing PGD.

Table 3. Table showing the number of reciprocal and Robertsonian translocation carriers, number of biopsied embryos, and number of balanced embryos

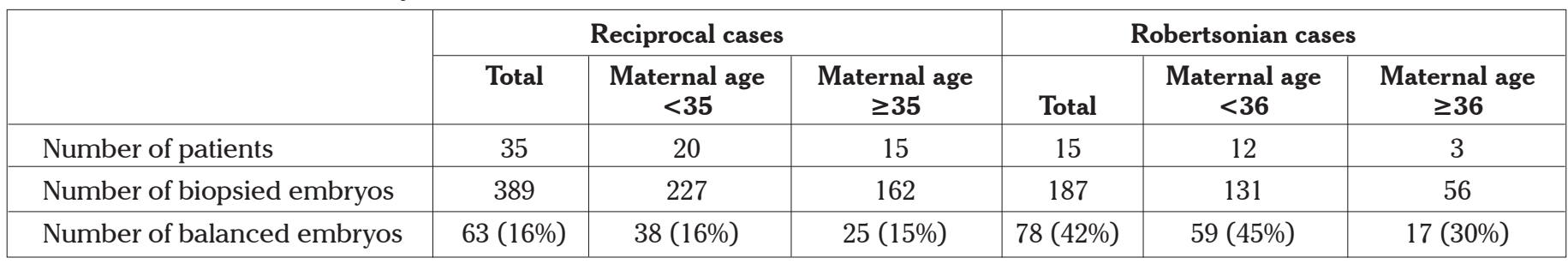


Ethics Committee Approval: Ethics committee approval was received for this study from the ethics committee (Approval number: BSG0020).

Informed Consent: Written informed consent was obtained from patients who participated in this study.

Peer-review: Externally peer-reviewed.

Author Contributions: Concept - P.T.; Design - P.T.; Supervision - P.T., M.B.; Materials - P.T., M.G., N.F.; Data Collection and/ or Processing - P.T., M.G.; Analysis and/or Interpretation - P.T.; Literature Review - P.T., N.F.; Writer - P.T.; Critical Review - P.T., M.G., N.F., M.B.

Conflict of Interest: No conflict of interest was declared by the authors.

Financial Disclosure: The authors declared that this study has received no financial support.

\section{References}

1. Simopoulou M, Harper JC, Fragouli E, Mantzouratou A, Speyer BE, Serhal P, et al. Preimplantation genetic diagnosis of chromosome abnormalities: implications from the outcome for couples with chromosomal rearrangements. Prenat Diagn 2003; 23: 652-62. [CrossRef]

2. Jacobs PA, Melville M, Ratcliffe S, Keay AJ, Syme J. A cytogenetic survey of 11,680 newborn infants. Ann Hum Genet 1974; 37: 359-76. [CrossRef]

3. Traversa MV, Carey L, Leigh D. A molecular strategy for routine preimplantation genetic diagnosis in both reciprocal and Robertsonian translocation carriers. Mol Hum Reprod 2010; 16: 329-37. [CrossRef]

4. Ford CE, Clegg HM. Reciprocal translocations. Br Med Bull 1969; 25: 110-4.

5. Rubio C, Bellver J, Rodrigo L, Bosch E, Mercader A, Vidal C, et al. Preimplantation genetic screening using fluorescence in situ hybridization in patients with repetitive implantation failure and advanced maternal age: two randomized trials. Fertil Steril 2013; 99: 1400-7. [CrossRef]

6. Mantzouratou A, Mania A, Fragouli E, Xanthopoulou L, Tashkandi S, Fordham K, et al. Variable aneuploidy mechanisms in embryos from couples with poor reproductive histories undergoing preimplantation genetic screening. Hum Reprod 2007; 22: 1844-53. [CrossRef]

7. Ulug U, Turan E, Tosun SB, Erden HF, Bahceci M. Comparison of preovulatory follicular concentrations of epidermal growth factor, insulin-like growth factor-I, and inhibins A and B in women undergoing assisted conception treatment with gonadotropin-releasing hormone (GnRH) agonists and GnRH antagonists. Fertil Steril 2007; 87: 995-8. [CrossRef]
8. Xanthopoulou L, Mantzouratou A, Mania A, Ghevaria H, Ghebo C, Serhal $\mathrm{P}$, et al. When is old too old for preimplantation genetic diagnosis for reciprocal translocations? Prenat Diagn 2011; 31: 1002-6. [CrossRef]

9. Munné S, Magli C, Bahce M, Fung J, Legator M, Morrison L, et al. Preimplantation diagnosis of the aneuploidies most commonly found in spontaneous abortions and live births: XY, 13, 14, 15, 16, 18, 21, 22. Prenat Diagn 1998; 18: 1459-66. [CrossRef]

10. Márquez C, Sandalinas M, Bahce M, Alikani M, Munné S. Chromosome abnormalities in 1255 cleavage-stage human embryos. Reprod Biomed Online 2000; 1: 17-26. [CrossRef]

11. Egozcue J. Preimplantation diagnosis in older patients. Of course not. Hum Reprod 1996; 11: 2077-8. [CrossRef]

12. Vidal F, Gimenez C, Rubio C, Simon C, Pellicer A, Santalo J, et al. FISH preimplantation diagnosis of chromosome aneuploidy in recurrent pregnancy wastage. J Assist Reprod Genet 1998; 15: 310-3. [CrossRef]

13. Alfarawati S, Fragouli E, Colls P, Wells D. Embryos of robertsonian translocation carriers exhibit a mitotic interchromosomal effect that enhances genetic instability during early development. PLoS Genet 2012; 8: e1003025. [CrossRef]

14. Anton E, Vidal F, Blanco J. Reciprocal translocations: tracing their meiotic behavior. Genet Med 2008; 10: 730-8. [CrossRef]

15. Anton E, Blanco J, Vidal F. Meiotic behavior of three D;G Robertsonian translocations: segregation and interchromosomal effect. J Hum Genet 2010; 55: 541-5. [CrossRef]

16. Guichaoua MR, Quack B, Speed RM, Noel B, Chandley AC, Luciani JM. Infertility in human males with autosomal translocations: meiotic study of a 14;22 Robertsonian translocation. Hum Genet 1990; 86: 162-6. [CrossRef]

17. Lejeune J. Autosomal Disorders. Pediatrics 1963; 32: 326-37.

18. Tulay P, Gultomruk M, Findikli N, Yagmur E, Bahceci M. Is the interchromosomal effect present in embryos derived from Robertsonian and reciprocal translocation carriers particularly focusing on chromosome 10 rearrangements? Zygote 2015; 23 : 908-15. [CrossRef]

19. Scriven PN, Flinter FA, Khalaf Y, Lashwood A, Mackie Ogilvie C. Benefits and drawbacks of preimplantation genetic diagnosis (PGD) for reciprocal translocations: lessons from a prospective cohort study. Eur J Hum Genet 2013; 21: 1035-41. [CrossRef]

20. Van Echten-Arends J, Coonen E, Reuters B, Suijkerbuijk RF, Dul EC, Land JA, et al. Preimplantation genetic diagnosis for X;autosome translocations: lessons from a case of misdiagnosis. Hum Reprod 2013; 28: 3141-5. [CrossRef]

21. Tulay P, Gultomruk M, Findikli N, Bahceci M. Poor embryo development and preimplantation genetic diagnosis outcomes of translocations involving chromosome 10: Do we blame genetics? Zygote 2015; 23: 778-84. [CrossRef] 